\title{
Application of an emittance adapter to increase photon flux density on a synchrotron radiation beam line
}

\author{
Pascale Brunelle, ${ }^{1,}$ Konstantin Klementiev, ${ }^{2}$ Jose Miguel Luque-Raigon, ${ }^{1}$ Olivier Marcouille, ${ }^{1}$ \\ Thierry Moreno, ${ }^{1}$ and Ryutaro Nagaoka ${ }^{1}$ \\ ${ }^{1}$ Synchrotron SOLEIL, Saint-Aubin B.P. 48, 91192 Gif sur Yvette CEDEX, France \\ ${ }^{2}$ MAX IV Laboratory, Lund University, P.O. Box 118, SE-221 00 Lund, Sweden
}

(Received 23 October 2018; published 20 June 2019)

\begin{abstract}
The application of the Chao-Raimondi emittance adapter to the SOLEIL storage ring is studied theoretically in terms of linear and nonlinear beam dynamics. This unique scheme recently proposed elsewhere using a pair of emittance adapters and a solenoid in between them allows a local conversion of an electron beam in a third generation light source to an ultralow emittance beam of the fourth generation. The study of potential benefits of the resultant synchrotron radiation is of crucial importance. Electron beam properties and magnetic fields inside the undulator solenoid are treated as inputs of the investigations. Characteristics of photon beams emitted by a round and small electron beam in an undulator are studied in detail on the basis of the near-field wave propagation method. The photon flux density gain, which is a figure of merit along with transverse coherence, is evaluated at the sample of two hard and soft x-ray beam lines existing at SOLEIL.
\end{abstract}

DOI: 10.1103/PhysRevAccelBeams.22.060702

\section{INTRODUCTION}

Since the advent of storage rings dedicated to synchrotron radiation (SR), there has been explosive progress in the material and biological science. Indeed, a large number of the so-called third generation light sources (3GLSs) have been constructed in the past couple of decades throughout the world, which are distinguished from the former generation rings by the use of insertion devices (IDs) such as undulators and wigglers, as the primary sources of SR, installed in a large number of free straight sections made available in the ring structure. The principal figure of merit for these storage ring-based light sources is the brilliance, which measures the number of photons emitted per unit surface, unit solid angle, second within $0.1 \%$ bandwidth of the concerned photon energy. Since the brilliance depends inversely proportional to the product of the two transverse electron beam emittances, endless efforts have been made across the accelerator community to design storage rings that store lower emittance electron beams.

While most 3GLSs operate today with electron beams having the horizontal emittance in the order of few nanometer radian ( $\mathrm{nm} \mathrm{rad}$ ), there has been a breakthrough

"pascale.brunelle@synchrotron-soleil.fr

Published by the American Physical Society under the terms of the Creative Commons Attribution 4.0 International license. Further distribution of this work must maintain attribution to the author(s) and the published article's title, journal citation, and DOI. in the accelerator technology over the past years to achieve a significant reduction in the horizontal emittance, of reaching a few hundreds of picometers radian ( $\mathrm{pm} \mathrm{rad}$ ) range or even lower. Since the attained horizontal emittance is already lower than the intrinsic diffraction-limited emittance of a radiated photon beam over a wide range of photon energy of interest, typically below the soft x ray, these new generation light sources are often called diffraction limited storage rings (DLSRs) or 4GLSs. Today there are two DLSRs constructed on a green field and two 3GLSs that are being upgraded to DLSRs [1]. In addition to which, many existing 3GLSs including SOLEIL are considering an upgrade to DLSRs.

Besides the technical challenge of converting an existing 3GLS to a DLSR, such a major upgrade of a storage ring is obviously an operation that cannot be carried out with ease as it would require a huge amount of budget as well as a long shutdown of the entire machine of the order of 1 to 2 years. It was under the consideration of these facts that a unique innovative scheme was proposed by Chao and Raimondi to convert locally a 3GLS to a DLSR with a set of what are called the emittance adapters and a solenoid in one of the available straight sections [2]. As it shall be explained in more detail below, the scheme allows a flat electron beam with a small vertical emittance to be converted locally to a round beam with very small horizontal and vertical emittances. Since at SOLEIL there is one $12 \mathrm{~m}$ long straight section still available, which is considered long enough to accommodate all the required elements above including an ID, a project was launched to 
study the technical feasibility of the emittance adapter scheme, and in particular its effective benefit on a beam line in terms of photon flux density.

The value of the electron beam emittance in reality is merely a figure of merit that scales the brilliance as mentioned above. What more directly impacts the performance of a beam line is the degree of matching between the distributions of an electron beam and a photon beam in the transverse phase space, i.e., the convolution of the two distributions. As revisited further later, the concerned adapter scheme generates an ultralow emittance electron beam by suppressing drastically its transverse divergence. The last is clearly a potential source of performance deterioration, which has already been pointed out elsewhere and investigated to some depth [3]. The main goal of the present paper is to make a detailed numerical and theoretical study of the electron and photon beams created by this scheme, by developing and applying a set of numerical tools to specific beam line configurations including those existing at SOLEIL. The notion of diffraction limited photon emittance is therefore fundamental in matching the electron and photon beams [4]. Given the diffraction limited photon emittance, the entire photon beam is then constructed by convoluting the former with the ensemble of electrons, by taking into account their distributions, which are usually Gaussian, in transverse position, angle as well as energy. In this framework, the properties of SR emitted by one single electron with a predefined trajectory should first be well characterized.

In this paper, we shall carry out a detailed calculation of wave propagation of SR emitted by an ultralow emittance electron beam created via a local emittance adapter as mentioned earlier to evaluate the effectiveness of the latter scheme. In particular, the properties of the resultant photon beam such as the flux density and transverse coherence shall be studied at different locations of the photon beam line such as at the diaphragm and the sample. The paper is organized as follows: in Sec. II we shall briefly introduce the emittance adapter scheme using a solenoid developed by Chao and Raimondi. Then in Sec. III we shall describe the application of this scheme in one long straight section of the SOLEIL storage ring, in terms of electron beam dynamics. In Sec. IV we shall present the benefit of this scheme on the photon flux density for two distinct beam lines, one soft $\mathrm{x}$ ray and the other hard $\mathrm{x}$ ray, existing at SOLEIL. A summary shall then be given in Sec. V.

The authors would like to mention that part of the results presented in this paper was obtained in the frame of the collaboration between SOLEIL and MAX IV (Sweden) laboratories. A Franco-Swedish team has been especially formed, composed of experts in beam line optics, synchrotron radiation experts and accelerator physicists to study the usefulness of the Chao-Raimondi adapter on a 3GLS. The collaboration was concluded by an international mini-workshop dedicated to "Round Beams" organized at SOLEIL on 14-15 June 2017, with a unique unprecedented attempt of bringing together, photon beam line scientists, accelerator physicists and experts in photon beam line optics and synchrotron radiation [5].

\section{LOCAL CONVERSION OF ELECTRON BEAM EMITTANCE TO ULTRALOW DIFFRACTION LIMITED VALUE WITH EMITTANCE ADAPTERS AND A SOLENOID}

For the sake of completeness, we describe synthetically the scheme developed by Chao and Raimondi hereafter that locally converts a flat electron beam having a horizontal emittance of the order of a few nm rad to a small and round beam whose two transverse emittances are diffraction limited for photon wavelengths in the range of general interest. Both the technique and the theory of beam manipulations from flat to round and vice versa are well established and have been applied to a wide range of accelerators: storage rings and free electron lasers [6] and high energy physics colliders [7]. There are three basic reasons for which the application to 3GLSs was proposed by Chao and Raimondi: First, the total conversion of a $3 \mathrm{GLS}$ ring to a DLSR would require both huge budgetary and technical efforts, where the magnet lattice design is expected to be particularly challenging in many aspects, while the proposed scheme allows a local conversion of a 3 GLS to a DLSR as already mentioned. Second, due to the nonzero longitudinal magnetic field component of a solenoid used in the scheme, there is a split between the canonical momenta $p_{x}$ and $p_{z}$ from the mechanical momenta $x^{\prime}(=d x / d s)$ and $z^{\prime}(=d z / d s)$. Here, conventionally $s$ denotes the longitudinal coordinate along the electron trajectory, $x$ the horizontal and $z$ the vertical coordinates. Thus, while neither the skew quadrupoles that are used for the flat to round conversions nor the solenoid are capable of varying the four-dimensional transverse emittance of an electron beam due to the symplecticity of their transformations, the apparent emittances determined by the mechanical momenta may be altered. Specifically, the proposed scheme establishes the following relation inside the solenoid:

$$
\begin{aligned}
& x^{\prime}=p_{x}-k_{s} z / 2 \\
& z^{\prime}=p_{z}+k_{s} x / 2,
\end{aligned}
$$

where $k_{s}=B_{s} /(B \rho)_{0}, B_{s}$ is the magnetic field of the solenoid and $(B \rho)_{0}$ is the magnetic rigidity of the electron beam. The matching between the adapters and the solenoid is made in such a way to have the two terms on the right sides of Eq. (1) cancel each other. This manipulation is justified upon the fact that the SR brilliance depends on the product of the two mechanical emittances of an electron beam. It follows that the mechanical or the apparent emittance of an electron beam in the solenoid is then given approximately by 


$$
\left(\varepsilon_{x}\right)_{\text {apparent }}=\left(\varepsilon_{z}\right)_{\text {apparent }} \approx \sqrt{\left(\varepsilon_{x} \varepsilon_{z}\right)_{\text {ring }}},
$$

where $\left(\varepsilon_{x}\right)_{\text {ring }}$ and $\left(\varepsilon_{z}\right)_{\text {ring }}$ denote respectively the original horizontal and vertical emittances of the electron beam defined by the rest of the ring. The last relation brings us to the third argument of Chao and Raimondi that $\left(\varepsilon_{x}\right)_{\text {apparent }}$ can be made significantly low by reducing $\left(\varepsilon_{z}\right)_{\text {ring }}$, namely the so-called emittance coupling of the ring, which is done routinely in many of the exiting $3 \mathrm{GLSs}$ even to the extent of below $0.1 \%$. Figure 1 shows that for the case of SOLEIL, the $4 \mathrm{~nm}$ rad ring emittance can be transformed in a $398 \mathrm{pm}$ rad local emittance for a $1 \%$ emittance coupling.

The developed emittance adapter scheme consists of two groups of skew quadrupoles and a solenoid in between. While the first group converts a flat electron beam to round at the entrance of the adapter section, the second one brings it back to flat at the exit. Each group is composed at least of three skew quadrupoles. The standard matching condition requires the beta functions to be equal $\left(\beta_{x}=\beta_{z}\right)$ and alpha functions to be zero $\left(\alpha_{x}=\alpha_{z}=0\right)$ at the entrance and the exit of the flat to round adapter, with the phase advance difference of $\pi / 2$, between horizontal and vertical planes, across the section $\left(\Delta \phi_{x}=\Delta \phi_{z}+\pi / 2\right)$. The condition $k_{s}=2 / \beta_{x}$ for the solenoid field then guarantees the round beam to stay constant (or laminar) across the solenoid section. The round to flat converter at the exit takes the complete mirror symmetric structure to the former, which makes the emittance adapter completely transparent to the rest of the ring in terms of linear optics.

It must be noted that the round beam in the solenoid has the transverse beam size of $\sigma_{x}=\sigma_{z}=\sqrt{\left(\varepsilon_{x}+\varepsilon_{z}\right) / k_{s}}$ and the divergence of $\sigma_{x}^{\prime}=\sigma_{z}^{\prime}=\sqrt{\varepsilon_{z} k_{s}}$. Namely, even though its apparent emittance can be made significantly small

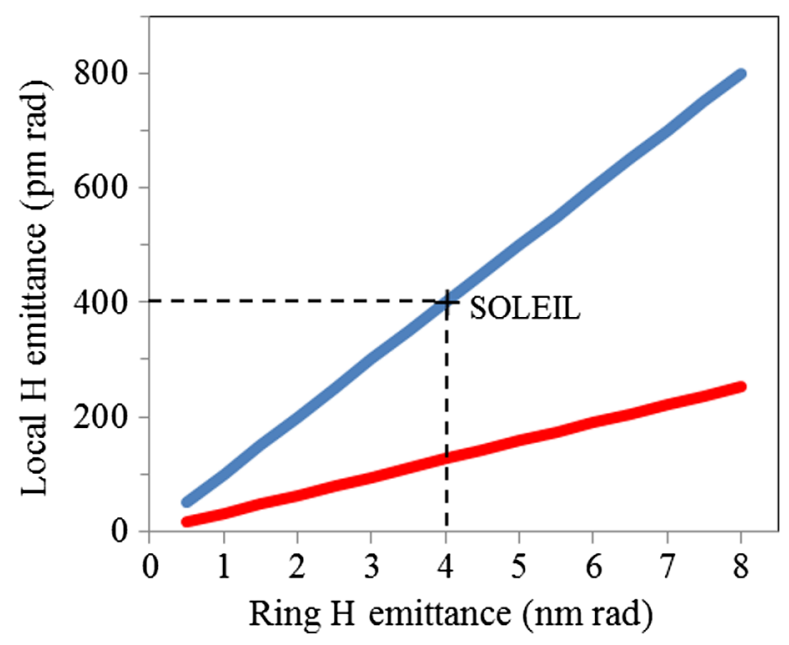

FIG. 1. Apparent local horizontal $(H)$ emittance attained with the Chao-Raimondi scheme for the SOLEIL case as a function of the original horizontal $(H)$ ring emittance and the emittance coupling: $1 \%$ (blue) and $0.1 \%$ (red). according to Eq. (2), the smallness is achieved principally via its reduced beam divergence and the beam size may remain relatively large. This particular feature of the round beam created by the emittance adapter is one of the main scopes of our investigation with respect to the resultant SR generated by the electron beam. The above relation indicates that the reduction of the round beam size scales with the square root of the solenoid field value.

Lastly, in order to have the SR from the obtained round beam, an undulator must be placed inside the solenoid. Although this may represent another huge technical challenge to be confronted, certain engineering studies have already been made elsewhere by insertion device experts, as a staggered undulator was proposed by Chavanne and others [3]. Naturally there are also a number of beam dynamics issues to be studied under the presence of both solenoid and undulator fields, among which, the impact of the solenoid field on the electron beam orbit inside an undulator is studied in the present paper (Sec. IV B).

\section{APPLICATION TO ONE LONG STRAIGHT SECTION AT SOLEIL}

The $4 \mathrm{~nm}$ rad emittance lattice of the SOLEIL storage ring possesses fourfold symmetry with a 12-m-long straight section in between super periods. As one long straight section (SDL09) remains free, studies began to prepare for a possible experimental demonstration of the efficiency of the emittance adapter to create a small and round electron beam on a $3 \mathrm{GLS}$. The original idea was to perform the demonstration with no cost, with the use of equipment available at SOLEIL or elsewhere.

\section{A. Implementation scheme and optics}

The scheme consists in introducing six additional skew quadrupoles (SQs) shared in two triplets located symmetrically with respect to the center of the long straight section (Fig. 2). The optimal arrangement takes into account the elements such as beam position monitors (BPMs), absorbers and vacuum valves existing at both ends of the section. There is nearly $90 \mathrm{~cm}$ of distance between the last quadrupole $(\mathrm{Q})$ of quadrupole triplet of the double bend cell and the first skew quadrupole to place the elements mentioned above. Each SQ triplet has the total length of $3 \mathrm{~m}$. The central section between the two SQ triplets is $4.23 \mathrm{~m}$ long, which is long enough to install a 2-m-long undulator combined with a solenoid. The photon beam (which is necessary to prove that the electron beam is round) should be created by a $60 \mathrm{~mm}$ period Apple-II type undulator available at SOLEIL. The skew quadrupoles were assumed to be substituted with unused quadrupoles provided by the ESRF that could be transformed in skew quadrupoles by rotating them with a new girder.

The necessary modification of the ring optics was made locally (Fig. 2) in the optics currently applied during the 


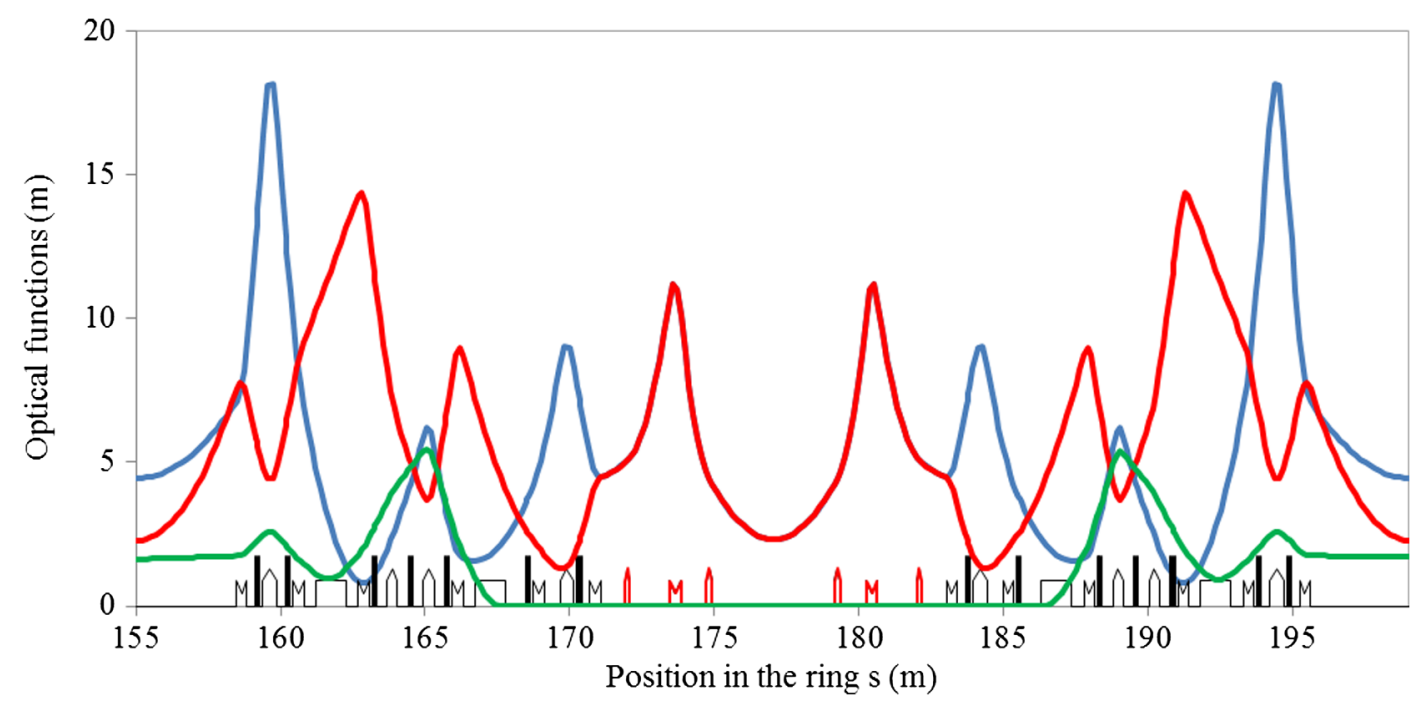

FIG. 2. Optical functions in the SDL09 straight section when the six additional skew quadrupoles are turned on. Magnetic structure: additional skew quadrupoles (up and down empty red arrows), dipoles (empty black rectangles), quadrupoles (up and down empty black arrows), sextupoles (black bars). Optical functions: horizontal beta (blue), vertical beta (red) and dispersion $\times 10$ (green).

operation to have the operational advantage in passing from the nominal mode to the other.

The six skew quadrupoles and all ten quadrupoles in the adjacent double bend cells were used in the matching. The horizontal dispersion was locally matched to zero across SDL09. The length of the skew quadrupoles was optimized in order to reach acceptable gradients below $40 \mathrm{~T} \mathrm{~m}^{-1}$. However, some normal quadrupoles of each adjacent cell reached their gradient limit of $20 \mathrm{~T} \mathrm{~m}^{-1}$. In the solution without a solenoid, betatron functions are equal and their derivatives are set to zero at the center of the straight with a phase advance difference of 90 degrees between the two planes in between the entrance of the skew quadrupole triplet and the center of the straight. Tunes are then adjusted to 18.176 and 10.234 (nominal values) using two quadrupole families, which only slightly destroys the above round beam adaptation without affecting the beam dynamics.

\section{B. Optimization of the vacuum chamber aperture to assure good injection efficiency}

It turned out, with the local matching as described above, that it is not possible to keep good injection efficiency with the present aperture of the vacuum chambers because of significant dynamic aperture reduction in both horizontal and vertical planes. The minimum required aperture of the vacuum chamber was therefore optimized both in the horizontal and vertical planes to reach a comfortably large on-momentum dynamic aperture (Fig. 3). The total horizontal aperture was kept to the nominal value of $56 \mathrm{~mm}$. Due to the full coupling at this location, the total vertical aperture has to be increased from 14 (nominal value) to $24 \mathrm{~mm}$ outside the undulator, at entry and exit of the straight section. This allows minimizing the loss of particles and avoiding any damage of the undulator magnets.
The 14-mm-vertical aperture in the undulator leads to a minimum magnetic gap of $19.5 \mathrm{~mm}$ instead of the usual $15.5 \mathrm{~mm}$ value for the present SOLEIL Apple-II type undulators. Four tapers have to be added in the straight section to accommodate the different vertical chamber apertures. To save some space around the undulator, it was decided to link the two vacuum chambers just before (after) the third (fourth) skew quadrupole. Calculations have shown that the tapering length has to be limited to
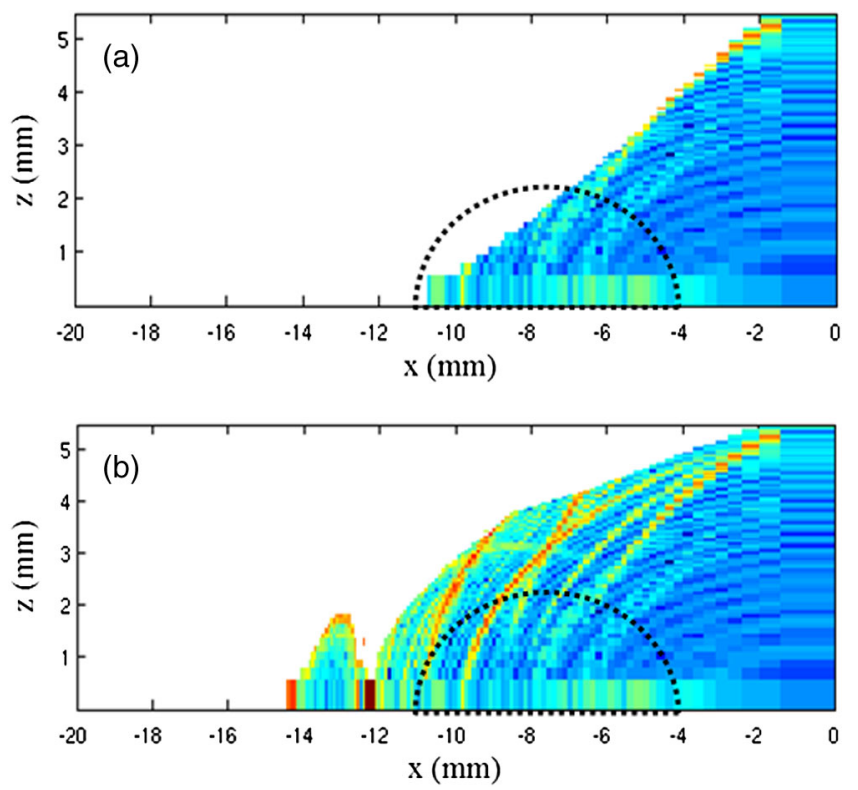

FIG. 3. Dynamic aperture computed by TRACY3 code [8] at the middle of the injection straight section using the nominal chamber dimensions (a) and the optimized chamber dimensions (b). The area defined by a black dashed curve represents the amplitude area to be stable for the injected beam. 
$0.2 \mathrm{~m}$ in order to keep a good dynamic aperture for injection.

Two BPMs are required close to the third and fourth skew quadrupoles in order to perform optics symmetrization with LOCO [9] and beam-based alignment of skew quadrupoles. The tuning of the undulator magnetic axis can be done when skew quadrupoles are off using the existing BPMs located at entry and exit of the straight section. In addition, it was verified that the photon flux emitted by the HU60 Apple-II type undulator at the gap of $19.5 \mathrm{~mm}$, instead of $15.5 \mathrm{~mm}$, is sufficient to obtain performant imaging using an x-ray pinhole camera installed in the tunnel. Finally, thanks to the magnetic measurements of the ESRF quadrupoles carried out [10], we tested the effect of the multipolar components of the six skew quadrupoles. No significant effect was found both on dynamic aperture and energy acceptance, even if the harmonic components would be multiplied by 10 .

\section{Expected performance of the emittance adapter and perspective}

The scheme consists in a local exchange of apparent horizontal and vertical emittance as mentioned in Sec. II. Due to the suppression of dispersion in the long straight section SDL09, the horizontal emittance of the ring is increased from 3.9 to $9.6 \mathrm{~nm}$ rad. With the nominal betatron coupling of $1 \%$, the emittance adapter can already provide locally, in the horizontal and vertical planes, an apparent emittance of $955 \mathrm{pm}$ rad, using a $10 \mathrm{~T}$ solenoid field, leading to electron beam sizes of $93 \mu \mathrm{m}$ in both planes and rather small divergence of $10 \mu \mathrm{rad}$ in both planes. It follows that the demonstration should be based more on the roundness of the electron beam than on its small sizes and divergences. Globally, the obtained results did not seem to indicate a critical issue in realizing the round beam according to the proposed emittance adapter schemes. However, there would be a significant reduction of the dynamic aperture if the scheme is combined with a vertically limited vacuum chamber. A temporary budget was evaluated for the equipment only, taking into account the possible contributions of ESRF and it turned out that this project could not be achieved at a low cost (even without solenoid) and that it significantly exceeds the level of expenditure that could be justified for the proof of principle alone. Prior to the experimental demonstration of the emittance adapter, therefore, the benefit of producing a round photon beam to increase the photon flux density on the sample of a beam line was studied.

\section{BENEFIT OF ROUND ELECTRON BEAM FOR PHOTON FLUX DENSITY: APPLICATION TO SOLEIL BEAM LINES}

In order to evaluate the impact of the Chao-Raimondi adapter, a comparison of the synchrotron radiation emitted by flat and round electron beams was performed for the soft x-ray SEXTANTS [11] and hard x-ray PROXIMA2 [12] beam lines, from the source to the experiment. The main results are presented in this section.

The SEXTANTS beam line is dedicated to the study of electronic and magnetic properties of the matter in the spectral range 50-1700 eV. In its coherent x-ray imaging mode (the one considered in this work), this beam line consists of a HU44 helical source undulator, a SX700 type monochromator [13] and a refocusing mirror and pinhole system to isolate the beam spatial coherence. PROXIMA2 (PX2) is a microfocusing biocrystallography beam line ranging in the spectral range $6-17 \mathrm{keV}$. The beam line is made of a canted U24 source undulator, a Si 111 channelcut monochromator and a pair of focusing mirrors in $\mathrm{KB}$ (Kirkpatrick Baez) arrangement [14]. Although PX2 is based on a canted U24 source, we have chosen to consider here a centered U20 undulator, which is a source configuration more widely used on hard $\mathrm{x}$-ray beam lines at SOLEIL.

For each considered case (electron beam configuration and beam line), the photon beam size (especially at the source location) has been carefully reconstructed through the near field wave propagation method of SRW [15] in order to evaluate the properties of coherence of the source emission. In this work, additional numerical libraries have been developed in order to allow including the electron beam energy spread into the SRW near field calculations. The beam propagation from the source to the sample was performed with the ray-tracing code SPOTX [16] after checking consistency with the wave propagation method at the diaphragm location. In the case of round electron beams, the calculation of the trajectories did not include the effect of the solenoid field.

The flux density calculation is based on a round electron beam generated by a Chao-Raimondi emittance adapter using a $10 \mathrm{~T}$ solenoid field. To simplify the comparison with the current situation, the $4 \mathrm{~nm}$ rad horizontal emittance of the ring is assumed to be preserved after optics matching. The electron beam size configurations used in the calculation are given in Table I for a $1 \%$ betatron coupling.

Note that some errors on the local tuning of the emittance adapter can propagate defaults elsewhere in the ring then impacting photon brightness for sensitive beam lines.

TABLE I. Electron beam size configurations used in the calculation.

\begin{tabular}{lccr}
\hline \hline Electron beam type & Flat SEXTANTS & Flat PX2 & Round \\
\hline Apparent emittance & $\ldots$ & $\ldots$ & 398 \\
$\quad(\mathrm{pm} \mathrm{rad})$ & & & \\
$\sigma_{x}(\mu \mathrm{m})$ & 212.2 & 224.5 & 60.3 \\
$\sigma_{x^{\prime}}(\mu \mathrm{rad})$ & 30.4 & 30.4 & 6.6 \\
$\sigma_{z}(\mu \mathrm{m})$ & 8.3 & 11.2 & 60.3 \\
$\sigma_{z^{\prime}}(\mu \mathrm{rad})$ & 3.7 & 4.3 & 6.6 \\
\hline \hline
\end{tabular}




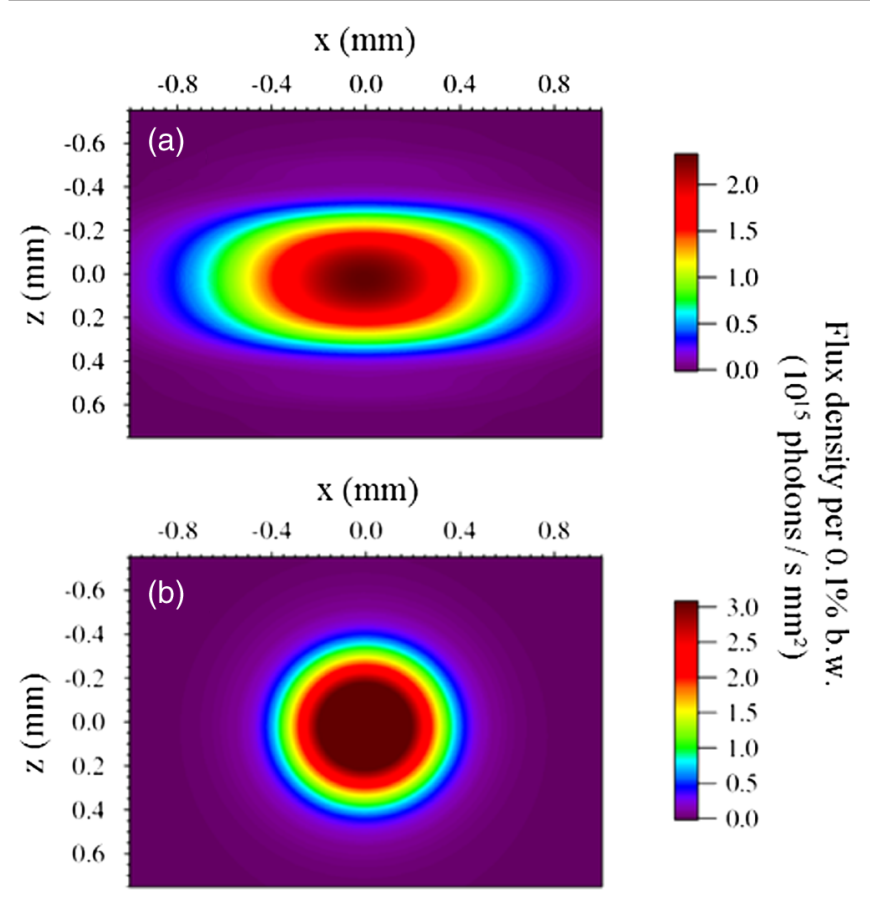

FIG. 4. Photon flux density reconstructed at the diaphragm $(s=10 \mathrm{~m}$ ) of the SEXTANTS beam line for flat (a) and round beam (b) configurations. Corresponding electron beam sizes and divergences are given in Table I. The photon energy is $780 \mathrm{eV}$.

Several kinds of errors have been identified: dipolar errors changing the electron beam position along the storage ring, coupling defaults modifying the vertical size of the electron beam. All these defaults should be locally or globally corrected via feedforward or feedback systems respectively.

\section{A. Photon flux density calculations}

All the calculations were performed for the $2.75 \mathrm{GeV}$ SOLEIL storage ring, a stored electron beam current of $500 \mathrm{~mA}$ and a natural energy spread of $1.01 \times 10^{-3}$.

The SEXTANTS simulations have been performed at the photon energy of $780 \mathrm{eV}$ (first harmonic) up to the entrance of the pinhole. The synchrotron radiation is emitted from the HU44 undulator working at a $20 \mathrm{~mm}$ gap in the right circular polarization mode. The magnetic field used in the calculation is the one obtained from magnetic measurements. The photon flux density is calculated at the source including electron beam energy spread, at the diaphragm $\left(s=10 \mathrm{~m}\right.$ with an aperture of $\left.2.0 \times 1.5 \mathrm{~mm}^{2} H \times V\right)$ (Fig. 4), and at the pinhole entrance taking into account the properties and defaults of all the existing beam line optical elements [11]. Results are summarized in Table II. The photon beam is fully incoherent in the horizontal plane and the degree of spatial coherence is $30 \%$ in the vertical plane, justifying the use of the ray-tracing approach. The benefit of a round beam, compared to a flat beam, in increasing the flux density at the sample is by a factor of 2 . The gain in flux density is strongly related to the reduction of the horizontal photon beam size at the source and could be further increased if radiation losses due to horizontal mirror slope errors are suppressed.

The PX2 simulations have been performed at the photon energy of $12.7 \mathrm{keV}$ (ninth harmonic) up to the sample (KB focal plane). Because of inevitable spectral and spatial distortions at high harmonic due to phase error, we consider first a perfect magnetic field calculated with the SRW code. The magnetic peak field of the U20 undulator is tuned to $0.9328 \mathrm{~T}$ to fit the photon energy of $12.7 \mathrm{keV}$ of the ninth harmonic. The photon flux is calculated at the source including electron beam energy spread, at the diaphragm $\left(s=15.73 \mathrm{~m}\right.$ with an aperture of $\left.1.6 \times 1.6 \mathrm{~mm}^{2}\right)$ and at the sample $(s=33.95 \mathrm{~m})$ taking into account the properties and defaults of all the existing beam line optical elements [12] (Fig. 5). Results are summarized in Table II. The source emission is incoherent for all the electron beam configurations. The photon beam is fully incoherent in both horizontal and vertical planes, justifying the use of the raytracing approach. The benefit of a round beam compared to a flat beam is a gain in flux density at the sample of a factor of 1.8. As previously noticed the gain in flux density is strongly related to the reduction of the horizontal photon beam size at the source and could be further increased if radiation losses due to horizontal mirror slope errors are

TABLE II. Photon flux and photon beam sizes at source, diaphragm and sample for the SEXTANTS and PX2 beam lines. Photon energies are respectively $780 \mathrm{eV}$ and $12.7 \mathrm{keV}$.

\begin{tabular}{lccccc}
\hline \hline & & \multicolumn{2}{c}{ SEXTANTS } & & \multicolumn{2}{c}{ PX2 } \\
\cline { 3 - 5 } & & Flat beam & Round beam & Flat beam & Round beam \\
\hline Source & Flux (photon/s $0.1 \%$ b.w.) & $1.24 \times 10^{15}$ & $1.25 \times 10^{15}$ & $2.14 \times 10^{14}$ & $1.59 \times 10^{14}$ \\
& $\sigma_{x} \times \sigma_{z}(\mathrm{rms})\left(\mu \mathrm{m}^{2}\right)$ & $211.5 \times 13.6$ & $61.7 \times 61.6$ & $204.9 \times 14.2$ & $60.5 \times 60.8$ \\
Diaphragm & Flux (photon/s 0.1\% b.w.) & $1.20 \times 10^{15}$ & $1.23 \times 10^{15}$ & $1.43 \times 10^{14}$ & $1.58 \times 10^{14}$ \\
& $\sigma_{x} \times \sigma_{z}(\mathrm{FWHM})\left(\mathrm{mm}^{2}\right)$ & $0.98 \times 0.46$ & $0.49 \times 0.49$ & $1.45 \times 0.68$ & $0.42 \times 0.66$ \\
Sample & Flux (photon $/ \mathrm{s})$ & $1.80 \times 10^{12}$ & $1.80 \times 10^{12}$ & $1.51 \times 10^{13}$ & $1.72 \times 10^{13}$ \\
& $\Delta \mathrm{E}(\mathrm{FWHM})(\mathrm{eV})$ & 0.077 & 0.200 & 3.72 & 3.68 \\
& $\sigma_{x} \times \sigma_{z}(\mathrm{FWHM})\left(\mu \mathrm{m}^{2}\right)$ & $151.0 \times 17.1$ & $67.5 \times 17.1$ & $14.8 \times 3.8$ & $4.7 \times 7.6$ \\
& Flux density (photon $\left./ \mathrm{s} \mathrm{mm}^{2}\right)$ & $7.0 \times 10^{14}$ & $1.4 \times 10^{15}$ & $2.68 \times 10^{17}$ & $4.81 \times 10^{17}$ \\
\hline \hline
\end{tabular}



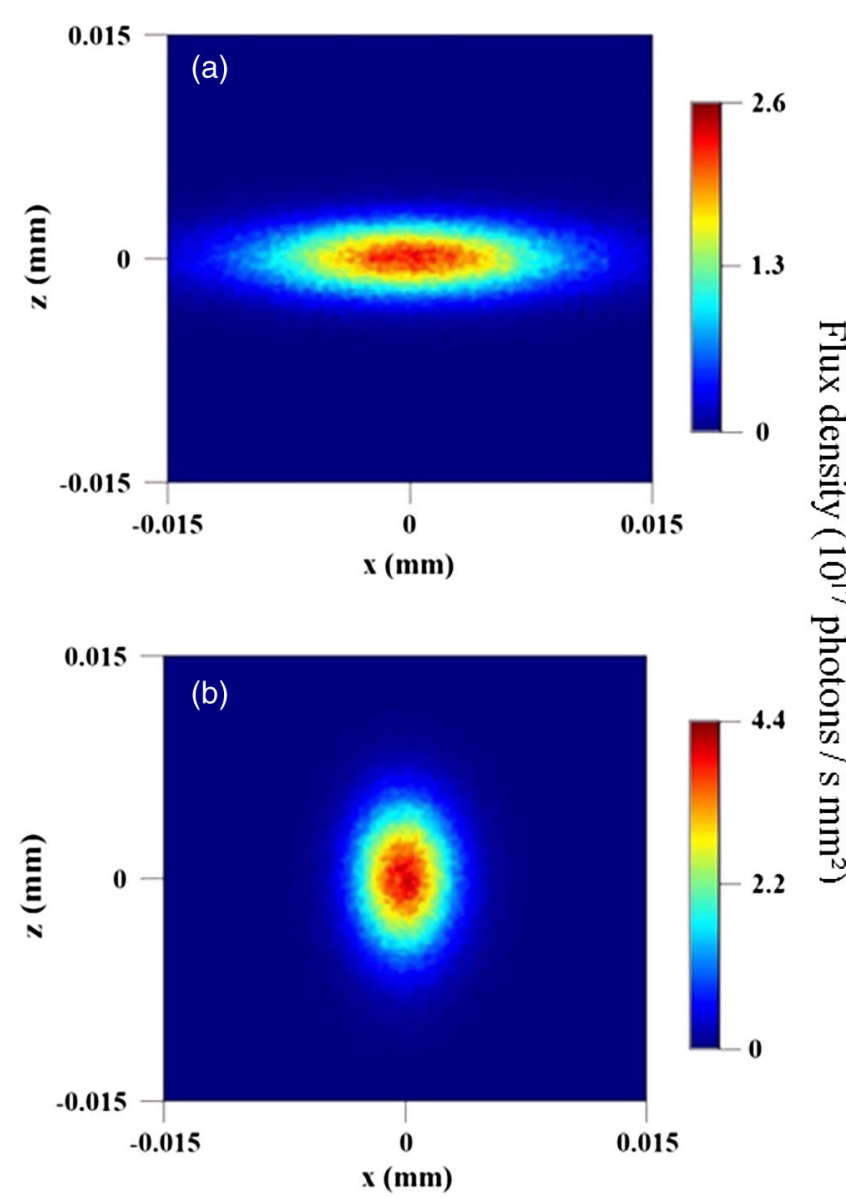

FIG. 5. Photon flux density reconstructed at the sample $(s=33.95 \mathrm{~m})$ of the PX2 beam line for flat (a) and round (b) beam configurations. Corresponding electron beam sizes and divergences are given in Table I. The photon energy is $12.7 \mathrm{keV}$.

minimized, for example by reducing the distance from KB mirrors to sample. We can point out that, in order to validate all the results obtained before, the use of a real magnetic field instead of a theoretical one as a source for the radiation was tested. Actually the real field given by Hall probe bench measurements presents small local variations of the peak amplitude (phase error) that may modify the emitted photon flux. The reduction of the total flux at the source and at the diaphragm due to phase error is the same for both flat and round electron beam configurations. As a consequence the gain in flux density obtained with a round beam remains unchanged when using a real magnetic field.

In Table II, it appears that the spectral resolution $\Delta \mathrm{E}$ is significantly degraded by the round beam configuration in the case of the SEXTANTS beam line while it remains almost unchanged for the PX2 case. This result is a direct consequence of the beam line configuration. The SEXTANTS spectral resolution is based on a SX700 grating monochromator [13] which is very sensitive to the vertical size of the source. The PX2 beam line is equipped with a channel-cut crystal monochromator facing the source and the spectral resolution only depends on the vertical photon beam divergence defined by the vertical beam line aperture (diaphragm and primary slits).

\section{B. Impact of the solenoidal field}

This section is devoted to evaluating the impact of the longitudinal solenoid field on the electron trajectory and on the spectral performances. The solenoid field is supposed to be ideal and the hard edge model is used. The U20 undulator described in Sec. IV is used for the calculation with a vertical magnetic field of $1 \mathrm{~T}$ (minimum gap of $5.5 \mathrm{~mm}$ ). A constant field $B_{1}$, generated by a solenoid, is artificially added in the longitudinal direction on top of the main vertical component produced by the undulator. The electrons exposed to the Lorentz force do not propagate anymore in the horizontal plane and tend to rotate around the longitudinal axis in traversing the undulator.

This effect is well predicted by the equations of motion:

$$
\frac{d \beta_{x}}{d s}=\frac{-e}{\gamma m_{0} c}\left(\beta_{z} B_{1}-\beta_{s} B_{z}\right) \quad \frac{d \beta_{z}}{d s}=\frac{e}{\gamma m_{0} c}\left(\beta_{x} B_{1}\right),
$$

where $\beta_{x, z}$ is the normalized particle speed respectively in the horizontal $(x)$ and vertical $(z)$ planes. The particle mass at rest, the Lorentz factor, the speed of light and the electron charge are respectively denoted as $m_{0}, \gamma, c$ and $e . B_{z}$ and $B_{1}$ are the magnetic fields generated respectively by the undulator and the solenoid.

The general trajectory parametrization [17] shows that the electron trajectory depends on the value of the solenoid field and on the angle of the trajectory at the entrance of the solenoid. Figure 6 shows trajectories, calculated by integrating twice the motion equations (first-order Runge-Kutta method), for different entrance angles with a reference system located at the undulator center $s=0$. In addition with the wiggles, the electron performs rotation around the propagation axis with amplitudes increasing with the entrance angle. If the electron enters the solenoid with a trajectory parallel to the axis, there is no effect of the longitudinal field. As can be seen in Fig. 6, the amplitude of the vertical trajectory is comparable to the vertical size of the electron beam. The impact on the focusing effect of the undulator cannot be considered as negligible, this was not evaluated. However, in operation, the vertical trajectory amplitude should be corrected and should become smaller than the beam size so that the solenoid field does not change the focusing effect of the undulator.

The corresponding photon spectrum calculated from the trajectories can be significantly modified when the effect of the solenoid field is taken into account (Fig. 7), especially when the entrance angle is larger than $10 \mu \mathrm{rad}$. This could be explained by the change of optical phase performed by the disturbed trajectory. As soon as the angle increases, the spectrum shifts toward lower energies and decreases in 


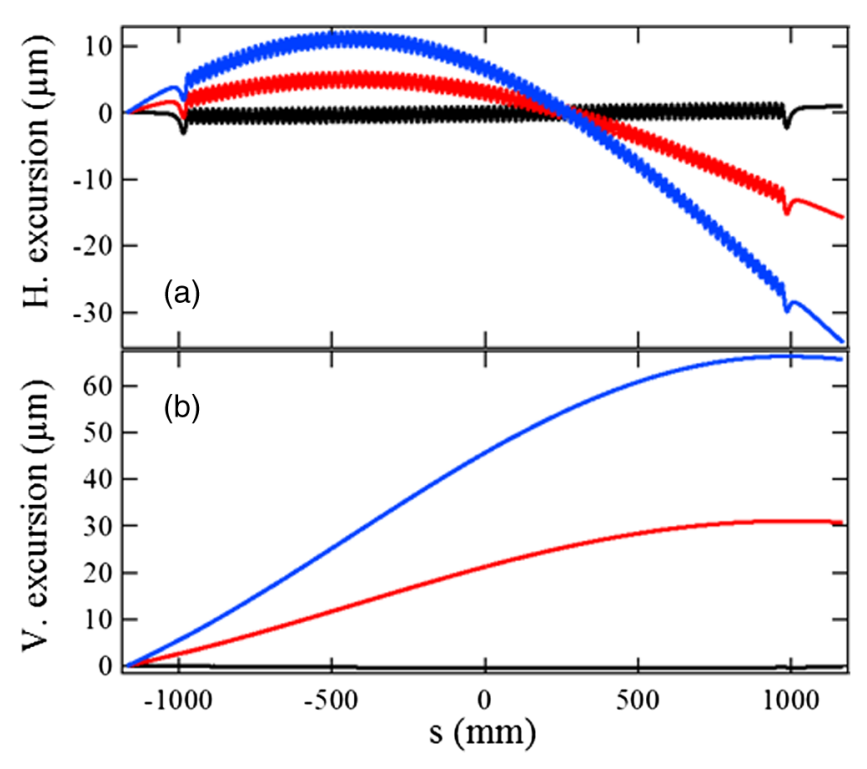

FIG. 6. Horizontal (a) and vertical (b) trajectory of one electron in the U20 undulator taking into account the $10 \mathrm{~T}$ solenoid field effect. Horizontal and vertical entrance angle are equal to $0 \mu \mathrm{rad}$ (black), $14 \mu \mathrm{rad}$ (red) and $30 \mu \mathrm{rad}$ (blue). For solenoid field values less or equal to $10 \mathrm{~T}$, the number of oscillations of the trajectory is much smaller than one, the trajectory is only bent.

amplitude. For high angle values, the spectrum is even degraded. However, the realistic electron beam is supposed to enter the undulator on axis with a zero entrance angle and a low angular divergence $\left(\sigma_{x^{\prime}, z^{\prime}}=6.6 \mu \mathrm{rad}\right.$ in the round beam configuration). Figure 8 shows that there is no significant effect on the flux density emitted by the electron beam. The slight change is due to the fact that some electrons in the beam enter the solenoid with nonzero angle because of the nonzero beam divergence. The change in electron trajectory due to the solenoid field does not modify the photon beam size at the diaphragm (Fig. 9) and generates a reduction of the flux by $7 \%$. Therefore, this

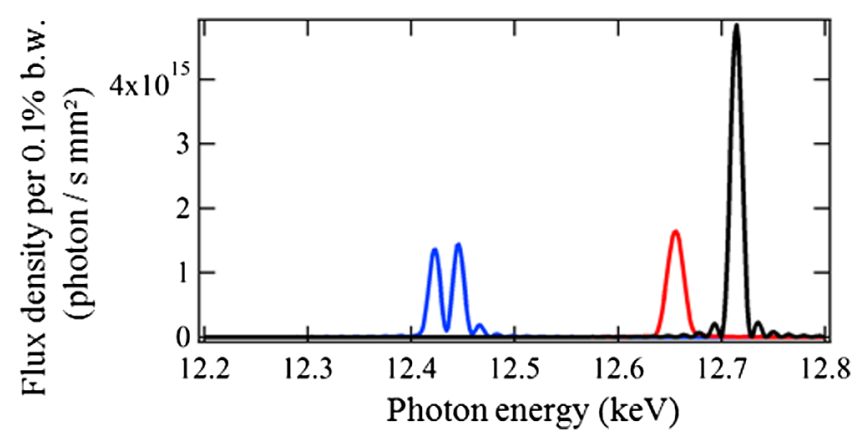

FIG. 7. On-axis photon spectra for the ninth energy harmonic emitted from the U20 undulator in the presence of the $10 \mathrm{~T}$ solenoid field taking into account the electron trajectories presented in Fig. 6. Horizontal and vertical entrance angle are equal to $0 \mu \mathrm{rad}$ (black), $14 \mu \mathrm{rad}$ (red) and $30 \mu \mathrm{rad}$ (blue).

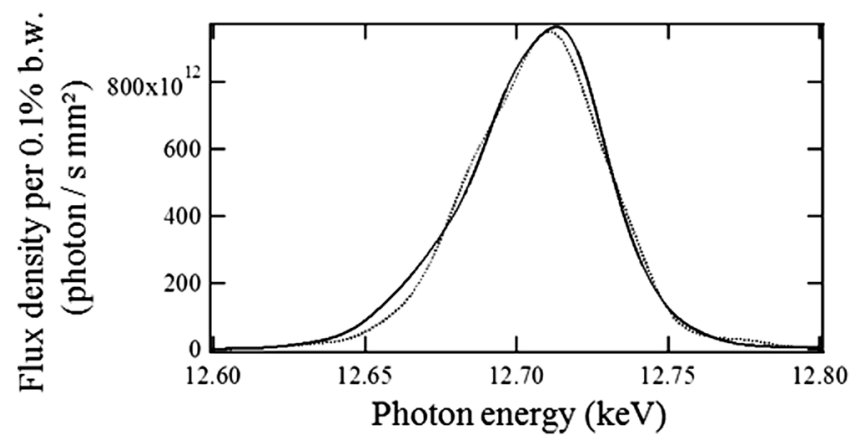

FIG. 8. Flux density radiated by the round electron beam (horizontal and vertical sizes and divergences are equal to $60.3 \mu \mathrm{m}$ and $6.6 \mu \mathrm{rad}$ respectively) taking into account the modified electron trajectories in the presence of $\left(B_{1}=10 \mathrm{~T}\right.$, solid line) and without $\left(B_{1}=0 \mathrm{~T}\right.$, dashed line) solenoid field effect. The electron beam enters the undulator on axis with zero angle.

effect of the solenoid field on the electron trajectory should be acceptable at the beam line sample where the photon beam appears focused. The conclusions obtained in Sec. IVA remain valid when the effect of the solenoid field is included in calculations. All the results were confirmed using the code XRT [18] developed by one of the authors (Klementiev).

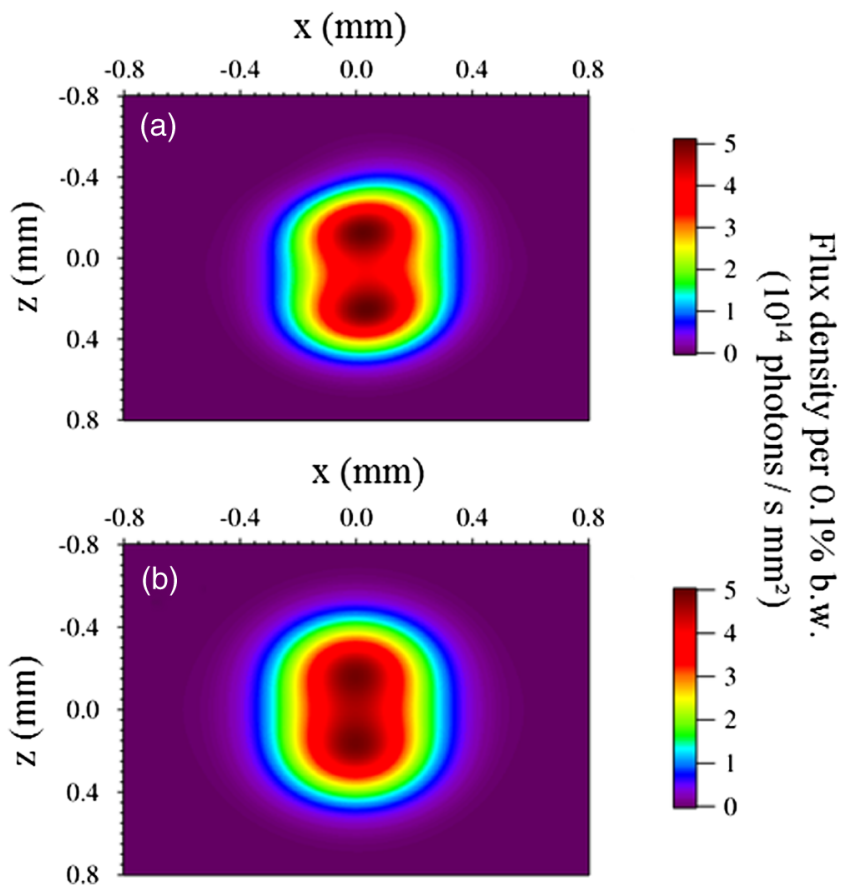

FIG. 9. Reconstruction of the photon beam size at the diaphragm of the PX2 beam line, corresponding to the U20 undulator emission, for round electron beam configuration (see Table II), taking into account the modified electron trajectories in the presence of the $10 \mathrm{~T}$ solenoid field (a) and without solenoid field effect (b). The photon energy is $12.7 \mathrm{keV}$. 


\section{SUMMARY}

The application of the Chao-Raimondi emittance adapter to the SOLEIL storage ring was studied theoretically in terms of linear and nonlinear beam dynamics. No critical issue was found in realizing the round beam according to the proposed emittance adapter scheme. However, some constituent elements needed such as the vacuum chamber would have had to be replaced, non-negligibly impacting the required budget. Prior to going to an experimental demonstration, therefore, it was decided to study first the benefit of a round and small electron beam in terms of flux density at the sample position of a beam line. Such an electron beam was evaluated to be attained by means of a local exchange of the apparent horizontal and vertical emittances à la Chao-Raimondi scheme with a solenoidal field of $10 \mathrm{~T}$. Applications were then made to two typical beam lines existing at SOLEIL using their present designs: one in the soft and the other in the hard x-ray range. In both cases it was demonstrated that the gain in flux density at the sample due to the use of a round and small electron beam is strongly related to the reduction of the horizontal photon beam size at the source. Nevertheless, the gain in flux density obtained at the sample turned out to be less than the reduction factor of the horizontal photon beam size at the source, as some losses are inherent to the present designs of the beam lines that are not adapted to small photon beams. Specifically, a gain in flux density at the sample of a factor of 2 was obtained for both beam lines with respect to the present configuration in the SOLEIL storage ring, while the reduction of the horizontal photon beam size at the source was by a factor of 3.4. The design of the beam line shall be improved in order to benefit from the electron beam size reduction at the source, for example in terms of horizontal mirror slope errors by reducing the distance between the mirrors and the sample. The effect of the solenoidal field on the electron trajectory was also studied, finding a negligible effect on the radiation performances thanks to the small electron beam divergences obtained with the ChaoRaimondi scheme. Finally it is worthwhile noting that round and small electron beams can be created by other means as well such as using the coupling resonance [19] or a Mobius scheme [20]. It must be stressed that all the procedures and tools developed in the present study can be applied to these different electron beam configurations.

\section{ACKNOWLEDGMENTS}

A part of this work was performed in the framework of collaboration between SOLEIL and MAX IV laboratories with constant encouragement of A. Nadji and C. Quitmann. The authors thank their colleagues from SOLEIL, in particular F. Polack (expert in photon beam line optics), F. Briquez, M.-E. Couprie (magnet and insertion device Group), W. Shepard (head of the PX2 beam line) and N. Jaouen (head of the SEXTANTS beam line) for their very helpful contributions. The authors thank warmly A. Chao and P. Raimondi for their original idea and for their constant support during the study. The authors are grateful for financial support through the Swedish Research Council funded Cooperation in the field of synchrotron light research between SOLEIL and MAX IV.

[1] R. Hettel, 4th generation storage ring light sources performance: goals and challenges, in Proceedings of the 6th DLSR Workshop, Berkeley, 2018, https://sites.google.com/ lbl.gov/als-dlsr2018-workshop/.

[2] A. W. Chao and P. Raimondi, Emittance adapter for a diffraction limited synchrotron radiation source, SLAC Report No. 14808, 2012.

[3] J. Chavanne, Some undulator photon beam properties in a flat to round electron beam insertion, ESRF Report No. 02-13/ID, 2013.

[4] H. Onuki and P. Elleaume, Undulators, Wigglers and Their Applications (Taylor \& Francis, New York, 2003).

[5] https://www.synchrotron-soleil.fr/en/events/mini-workshopround-beams.

[6] R. Brinkmann, Application of flat to round beam transformation for radiation sources, in Proceedings of the 8th European Particle Accelerator Conference, Paris, 2002 (EPS-IGA and CERN, Geneva, 2002), p. 653.

[7] A. Burov, S. Nagaitsev, A. Shemyakin, and Ya. Derbenev, Optical principles of beam transport for relativistic electron cooling, Phys. Rev. ST Accel. Beams 3, 094002 (2000).

[8] L. Nadolski and J. Zhang, TRACY3, SOLEIL version.

[9] G. Portmann, J. Safranek, and X. Huang, Matlab based LOCO, Report No. SLAC-PUB-14640, 2007.

[10] J. Chavanne (private communication).

[11] https://www.synchrotron-soleil.fr/fr/lignes-de-lumiere/ sextants.

[12] https://www.synchrotron-soleil.fr/fr/lignes-de-lumiere/ proxima-2a.

[13] H. Petersen, The plane grating and elliptical mirror: A new optical configuration for monochromators, Opt. Commun. 40, 402 (1982); The high energy plane grating monochromators at BESSY, Nucl. Instrum. Methods Phys. Res., Sect. A 246, 260 (1986).

[14] P. Kirkpatrick and A. V. Baez, Formation of optical images by x rays, J. Opt. Soc. Am. 38, 766 (1948).

[15] O. Chubar and P. Elleaume, Accurate and efficient computation of synchrotron radiation in the near field region, in Proceedings of the 6th European Particle Accelerator Conference, Stockholm, 1998 (IOP, London, 1998), p. 1177.

[16] T. Moreno and M. Idir, SPOTX a ray tracing software for X-ray optics, J. Phys. IV 11, Pr2-527 (2001).

[17] J. M. Luque-Raigon, SOLEIL Report No. AI-PM-RA-I0555, 2017.

[18] K. Klementiev and R. Chernikov, Powerful scriptable ray tracing package xrt, in Advances in Computational Methods for X-Ray Optics III, edited by M. Sanchez del Rio and O. Chubar, in Proceedings of SPIE, Vol. 9209 
(SPIE, Bellingham, WA, 2014), p. 92090A, https://doi.org/ 10.1117/12.2061400.

[19] P. Kuske, Creating round beams by linear coupling, Low Emittance Rings Workshop, Synchrotron SOLEIL, Gif sur Yvette, 2016, https://indico.cern.ch/event/574973/.
[20] M. Aiba, M. Ehrlichman, and A. Streun, Round beam operation in electron storage rings and generalisation of Mobius accelerator, in Proceedings of the IPAC2015 conference, Richmond, 2015 (JACoW, Geneva, Switzerland, 2015), p. 1716, https://doi.org/10.18429/JACoWIPAC2015-TUPJE045. 